\title{
Switching Patients with Type 1 Diabetes to Insulin Degludec from Other Basal Insulins: Real-World Data of Effectiveness and Safety
}

\author{
Paola Ponzani - Cesare Berra - Alessandra Di Lelio - Paola Del Sindaco • \\ Chiara Di Loreto · Francesco Reggiani - Giuseppe Lucisano • \\ Maria Chiara Rossi (D)
}

Received: August 8, 2019 / Published online: November 9, 2019

(c) The Author(s) 2019

\begin{abstract}
Introduction: Real-world evidence on the effectiveness and safety of insulin degludec (IDeg) in patients with diabetes is a priority. We have therefore evaluated the effectiveness and safety of IDeg, including impact on metabolic control, glycemic variability, weight gain and hypoglycemia, in patients with type 1 diabetes under routine clinical practice conditions.

Methods: This was an observational longitudinal multicenter study. A retrospective chart review of
\end{abstract}

Enhanced Digital Features To view enhanced digital features for this article go to https://doi.org/10.6084/ m9.figshare.10042994.

Electronic Supplementary Material The online version of this article (https://doi.org/10.1007/s13300019-00722-y) contains supplementary material, which is available to authorized users.

P. Ponzani

SSD Endocrinologia, Diabetologia e Malattie

Metaboliche, ASL3 Genovese, Genoa, Italy

C. Berra · F. Reggiani

Humanitas Research Institute, Rozzano, MI, Italy

A. Di Lelio · G. Lucisano · M. C. Rossi $(\bowtie)$

Center for Outcomes Research and Clinical

Epidemiology (CORESEARCH), Pescara, Italy

e-mail: rossi@coresearch.it

P. Del Sindaco · C. Di Loreto

Servizio di Diabetologia del Perugino, USL Umbria 1, Perugia, Italy all patients with type 1 diabetes who were switched from basal insulin to IDeg was performed, and temporal trends in clinical outcomes were assessed.

Results: Data obtained from 195 patients, with a median age of 42.8 [interquartile range (IQR) 24.6-56.4] years and a median diabetes duration of 16 (IQR 10.0-28) years, were analyzed. Median follow-up was 9.5 (IQR 7.7-11.3) months. Improvements were found in glycated hemoglobin $(-0.34 \% ; p<0.0001)$, fasting blood glucose $(-24.82 \mathrm{mg} / \mathrm{dL} ; \quad p<0.0001)$, postprandial glucose $(-17.23 \mathrm{mg} / \mathrm{dL} ; p=0.0009)$, glycemic variability as indicated by standard deviation of blood glucose $(-5.67 \mathrm{mg} / \mathrm{dL} ; p$ $<0.0001)$ and high blood glucose index $(-3.77 ; p<0.0001)$. Body weight and body mass index remained substantially stable during the follow-up $(-0.18 \mathrm{~kg} ; p=0.56$ and -0.12 ; $p=0.42$, respectively). Risk of nocturnal hypoglycemia decreased by $52 \%$ [incidence rate ratio 0.48 ; 95\% confidence interval (CI) $0.29-0.77$ ] and risk of total hypoglycemic episodes by $41 \%$ (incidence ratio 0.59; 95\% CI 0.45-0.83). Basal and short-acting insulin doses decreased by -1.4 and $-3.1 \mathrm{IU}$, respectively.

Conclusion: Switching patients with type 1 diabetes to IDeg from other basal insulins was associated with relevant improvements in metabolic control and glycemic variability without weight gain; the risk of hypoglycemic episodes also significantly declined. 
Funding: Novo Nordisk S.p.A. unconditional grant.

Keywords: Effectiveness; Glycemic variability; Hypoglycemia; Insulin degludec; Real-world data; Type 1 diabetes

\section{Key Summary Points}

Why carry out this study?

Ultra-long-acting insulin degludec (IDeg) is a recent therapeutic option for patients with diabetes requiring treatment with basal insulin.

In addition to experimental studies, realworld data are strongly required to obtain an overall picture of the effectiveness and safety of new drugs when used under routine clinical practice conditions.

\section{What did the study ask?}

What is the effectiveness and safety of IDeg in people with type 1 diabetes treated under routine clinical practice conditions?

\section{What was learned from the study?}

Switching patients with type 1 diabetes to IDeg from other basal insulins significantly improves glycemic control and reduces glycemic variability.

Use of IDeg is associated with a decrease in the risk of hypoglycemia and no weight gain.

These benefits were obtained despite a reduction in both basal and short-acting insulin doses during the follow-up.

\section{INTRODUCTION}

Degludec (IDeg) is an ultra-long-acting insulin recently introduced as a therapeutic option for patients with diabetes requiring treatment with basal insulin [1]. The efficacy and safety of IDeg have been extensively tested in the BEGIN ${ }^{\circledR}$ studies, a comprehensive research program involving patients with type 1 and type 2 diabetes (T1DM and T2DM, respectively) [2-4].

In the BEGIN Basal-Bolus Type 1 (BEGIN BBT1D) trial, subjects with T1DM were treated for 52 weeks with either IDeg or insulin glargine (IGlar). At the end of the study period, glycated hemoglobin (HbA1c) had decreased by $0.40 \%$, mean baseline fasting plasma glucose (FPG) had decreased by $1.3-7.8 \mathrm{mmol} / \mathrm{L}$ and mean weight had increased by $1.8 \mathrm{~kg}$ in those patients in the IDeg arm. Rates of overall confirmed hypoglycemia were similar in the IDeg and IGlar arms of the study [ 42.54 vs. 40.18 episodes per patient-year of exposure (PYE)]. The rate of nocturnal confirmed hypoglycemia was $25 \%$ lower in those receiving IDeg than in those treated with IGlar (4.41 vs. 5.86 episodes per PYE) [2].

In the BEGIN Flex T1 trial, the efficacy and safety of IDeg administered to subjects with T1DM once daily at varying times (IDeg forced flexible regimen [IDeg Forced-Flex arm]) was compared to IDeg and IGlar administered once daily at the same time each day. After 26 weeks of treatment, those subjects in the IDeg arms had lower HbA1c (IDeg Forced-Flex - 0.40\%) and (IDeg - 0.41\%) than those in the IGlar arm. FPG reductions were similar in both the IDeg Forced-Flex and IGlar arms, but higher in the IDeg arm $(-2.54 \mathrm{mmol} / \mathrm{L})$ than in the IDeg Forced-Flex arm $(-1.28 \mathrm{mmol} / \mathrm{L})$. Mean weight gain from baseline to week 26 and during the extension was modest, with no between-group differences at week 52 (IDeg Free-Flex $1.3 \mathrm{~kg}$; IGlar $1.9 \mathrm{~kg}$ ). Confirmed hypoglycemia rates were similar at weeks 26 and 52. Nocturnal confirmed hypoglycemia was lower in the IDeg Forced-Flex arm vs. the IDeg (37\%) and IGlar (40\%) arms at week 26 and 25\% lower with IDeg Free-Flex vs. IGlar at week 52 [3].

The BEGIN BBT1 study compared IDeg and insulin detemir (IDet), both administered as basal treatment once daily, in terms of efficacy and safety for the treatment of T1DM. After 26 weeks, HbA1c had decreased by $0.73 \%$ with IDeg, and FPG had decreased by $2.60 \mathrm{mmol} / \mathrm{L}$ with IDeg and by $0.62 \mathrm{mmol} / \mathrm{l}$ with IDet. Mean 
body weight increased from baseline to end of treatment with both IDeg $(1.5 \mathrm{~kg})$ and IDet $(0.4 \mathrm{~kg})$. The rate of confirmed hypoglycemia was similar in the IDeg and IDet study arms ( 45.83 vs. 45.69 episodes per PYE), while the rate of nocturnal confirmed hypoglycemia was lower in the IDeg arm than in the IDet arm (4.14 vs. 5.93 episodes per PYE) [4].

The results of the above-mentioned randomized clinical trials are consistent in showing that HbA1c (primary outcome) and FPG were reduced by IDeg to a similar degree as by IGlar and IDet, but with a lower risk of nocturnal confirmed hypoglycemia. The lower day-to-day variability of IDeg treatment is responsible for the more predictable effect and the more favorable safety profile of IDeg versus IGlar [5]. These findings suggest that IDeg represents an advance in diabetes therapy due to its positive impact on hypoglycemia and fear of hypoglycemia, two factors recognized as main barriers to the achievement of glucose targets and patient quality of life [6, 7]. Nevertheless, a slightly higher increase in body weight was found with IDeg versus IGlar and IDet.

Experimental studies provide important information. Real-world data are also important in providing relevant information on the effectiveness and safety of any new drug when used in routine clinical practice $[8,9]$. However, the impact of IDeg on metabolic control, hypoglycemia and body weight when used in real-world patient populations has seldom been investigated $[10,11]$. Therefore, additional realworld data are needed in order to support the effectiveness and safety of switching from other basal insulins to IDeg in patients with T1DM.

The overall aim of the study was to evaluate patterns of use and the effectiveness and safety of IDeg in routine clinical practice. The primary objective of the study was to evaluate whether the reduction in HbA1c observed in clinical trials could be attained in patients with T1D who switch to IDeg from other basal insulins during routine management in outpatient clinics. Secondary objectives included evaluation of the attainment of beneficial effects on blood glucose levels and variability, body weight and hypoglycemia under the same conditions.

\section{METHODS}

This was an observational, retrospective longitudinal study. A paper based on the same study and based on a cohort with T2DM has been published previously [12]; the methods used in this paper are similar to those reported in this earlier study. Consecutive patients attending four diabetes outpatient clinics in Italy who had started the treatment with IDeg under routine clinical practice conditions were included in the study. All treated patients were included to minimize the selection bias.

The date of starting IDeg therapy (T0) represented the baseline visit. Data on age, sex, body mass index (BMI), diabetes duration, diabetes-related complications and previous basal insulin treatments were collected as patient baseline characteristics. Chronic kidney disease was defined as an estimated glomerular filtration rate of $<60 \mathrm{~mL} / \mathrm{min}$ (CKD-EPI formula). Collected data were derived from the electronic medical records of each participating center maintained for the routine management of patients.

At the baseline visit (T0) and during the two subsequent follow-up visits (T1 and T2), information was collected on HbA1c, fasting blood glucose (FBG), post-prandial glucose (PPG), glycemic variability (i.e. standard deviation of mean blood glucose level), high blood glucose and low blood glucose indices (HBGI and LBGI, respectively), body weight, doses of basal and short-acting insulin and overall, nocturnal and severe hypoglycemia episodes. Visits were scheduled according to the routine clinical practice.

HbA1c levels were measured in the laboratories of the hospitals participating in the study. A standardized method [Diabetes Control and Complications Trial (DCCT)-aligned HbA1c method] was adopted by all laboratories. Blood glucose data were obtained from the downloads of glucose meters routinely used by the patients. Data on FBG, PPG, glycemic variability, and hypoglycemia events included all of the values recorded during the month before each visit. Overall hypoglycemia was defined as all blood glucose values $<70 \mathrm{mg} / \mathrm{dL}$, while severe 
hypoglycemia was defined as a hypoglycemia episode requiring assistance by a third person. Nocturnal hypoglycemia was defined as a blood glucose value of $<70 \mathrm{mg} / \mathrm{dL}$ recorded between midnight and $7.00 \mathrm{am}$.

All procedures followed were in accordance with the ethical standards of the responsible committee on human experimentation (institutional and national) and with the Helsinki Declaration of 1964, as revised in 2013. Informed consent was obtained from all patients for inclusion in the study. The study protocol was approved by the following Ethics Committees of the participating centers: Comitato Etico Regionale della Liguria, Genova (GE); Comitato Etico Indipendente Istituto Clinico Humanitas, Rozzano (MI); CER UMBRIA, Comitato Etico Regionale Umbria, Perugia (PG).

\section{Statistical Analysis}

Descriptive data were expressed as median and interquartile range (IQR) for continuous variables and as percentage for categorical variables. Trends over time in continuous endpoints (HbA1c, FBG, PPG, glycemic variability, weight, HBGI, LBGI, insulin dose) were assessed using longitudinal linear models for repeated measures. All longitudinal models took into account three time points (T0, T1, T2); only for insulin doses was an additional time point considered, namely $\mathrm{T}-1$, corresponding to the last prescribed dose of the previous basal insulin before the initiation of IDeg. To account for withinpatient correlation over time and unequal duration of follow-up, we used an unstructured correlation type for all longitudinal models. Results were expressed as estimated mean and estimated mean change from baseline with their 95\% confidence intervals (CIs). $p$ values of $<0.05$ were considered to be statistically significant.

Rates of hypoglycemic episodes were assessed during the 1 month preceding the IDeg start date (T0), during 1 month before the first follow-up visit (T1) and during 1 month before the second follow-up visit (T2). Incidence rates at
$\mathrm{T} 1$ versus $\mathrm{T} 0$ and at $\mathrm{T} 2$ versus $\mathrm{T} 0$ were compared using the Wilcoxon test.

All statistical analyses were performed with SAS software release 9.4 (SAS Institute, Cary, NC, USA).

\section{RESULTS}

Data on 195 patients with T1DM who started the treatment with IDeg between November 2014 and November 2015 were analyzed. The first follow-up visit (T1) was performed at a median of 4.2 (IQR 3.1-5.7) months after baseline (T0), while the second follow-up visit (T2) was performed at a median of 9.5 (IQR 7.7-11.3) months after T0. Information on data completeness at each visit is reported in Electronic Supplementary Material (ESM) Table S1.

Baseline characteristics overall and by treatment modality are reported in Table 1 . The median age of the study population was 42.8 (IQR 24.6-56.4) years, 41.5\% were men and median diabetes duration was 16 (IQR 10.0-28.0) years. At baseline, median BMI was 24.2 (IQR 21.9-27.5) $\mathrm{kg} / \mathrm{m}^{2}$, chronic kidney disease was present in $4.6 \%$ of the patients and $7.7 \%$ of patients had a history of cardiovascular events.

At the end of the observation period, improvements were found in HbA1c $(-0.34 \%$; $p<0.0001)$, FBG $(-24.82 \mathrm{mg} / \mathrm{dL} ; p<0.0001)$ and PPG $(-17.23 \mathrm{mg} / \mathrm{dl} ; p=0.001)$. A statistically significant decrease in the standard deviation of mean blood glucose $(-5.67 \mathrm{mg} / \mathrm{dl} ; p$ $<0.0001)$ and in the HBGI $(-3.77 ; p<0.0001)$ were found, while the LBGI remained unchanged. A not statistically significant decrease in average body weight was found $(-0.18 \mathrm{~kg}$; $p=0.56$ ) (Table 2).

Changes in IDeg and short-acting insulin doses at each visit were assessed. The dose of the previous basal insulin was also tested in the model (Table 3). At T-1, 13.6\% of patients were treated with IDetr once daily, $19.4 \%$ with IDet twice daily, $66.5 \%$ with IGlar U100 once daily and $0.5 \%$ with IGlar U100 twice daily.

The dose of basal insulin was progressively titrated from $\mathrm{T} 0$ to $\mathrm{T} 2$, reaching an average dose of 19.7 IU at T2. During the follow-up, basal and 
Table 1 Baseline characteristics of patients with type 1 diabetes mellitus participating in the study

\begin{tabular}{lc}
\hline $\begin{array}{l}\text { Baseline characteristics of total } \\
\text { patient population }(\boldsymbol{n}=\mathbf{1 9 5})\end{array}$ & Overall \\
\hline Age (years) & $42.8(\mathrm{IQR}$ \\
& $24.6-56.4)$ \\
Sex (\%) & 41.5 \\
Male & 58.5 \\
Female & $16.0(\mathrm{IQR}$ \\
Diabetes duration (years) & $10.0-28.0)$ \\
& $24.2(\mathrm{IQR}$ \\
Body mass index (kg/m $\left.{ }^{2}\right)$ & $21.9-27.5)$ \\
Chronic kidney disease (\%) & 4.6 \\
Previous cardiovascular event (\%) & 7.7 \\
Previous basal insulin therapy (\%) & \\
Detemir once daily & 13.6 \\
Detemir twice daily & 19.4 \\
Glargine once daily & 66.5 \\
Glargine twice daily & 0.5 \\
Lispro protamine & 0 \\
Pre-mix & 0 \\
\hline Dax &
\end{tabular}

Data are presented as the median and interquartile (IQR) range, or as a proportion (\%) of the total population

short-acting insulin doses decreased (estimated mean change from T-1) by -1.4 (95\% CI -2.1 to -0.7$)$ IU and by $-3.1(95 \% \mathrm{CI}-4.2$ to - 2.0) IU, respectively.

The incidence of overall, nocturnal and severe hypoglycemia decreased during the followup (Table 4). In terms of the relative risk of experiencing hypoglycemic episodes at T2 versus $\mathrm{T} 0$, the incidence rate ratio was 0.48 (95\% CI $0.29-0.77 ; p=0.004)$ for nocturnal episodes, 0.04 (95\% CI $0.001-1.43 ; p=0.08$ ) for severe episodes and 0.59 (95\% CI $0.45-0.83)$; $p=0.001)$ for total episodes.

Additional analyses on changes in HbA1c levels and rate of hypoglycemia during the follow-up stratified by previous basal insulin are reported in ESM Tables S2 and S3.

\section{DISCUSSION}

This article follows up on our previous publication based on the same study [12] focusing on the benefits of IDeg for treating T2DM and provides evidence on the impact of initiating IDeg therapy in patients with T1DM in the realworld setting.

Consistent with the results of the BEGIN studies on T1DM [2-4], our study confirms that IDeg provides statistically significant and clinically relevant improvements in metabolic control. In the BEGIN studies, HbA1c was improved by $0.4-0.7 \%$, while in our study, based on routinely collected data, HbA1c was reduced by $0.34 \%$. Interestingly, the positive effects on metabolic control were obtained despite a reduction in the total insulin dose. It can be speculated that these benefits could derive from the stable concentration of the drug, as suggested by pharmacodynamics and pharmacokinetics studies [5], rather than by an optimization of both basal and short-acting insulin doses.

In our study, statistically significant and clinically relevant reductions in the FBG $(-24.8 \mathrm{mg} / \mathrm{dL})$ and PPG $(-17.2 \mathrm{mg} / \mathrm{dL})$ levels and in glycemic variability (standard deviation of mean blood glucose $-5.7 \mathrm{mg} / \mathrm{dl}$; HBGI - 3.74) were also found following the initiation of IDeg. In addition, following the start of IDeg therapy, no severe hypoglycemic episodes were recorded and the incidence of overall hypoglycemia significantly decreased. Also, the risk of nocturnal episodes and total number of episodes of hypoglycemia significantly decreased, by 52 and $41 \%$, respectively. The risk of severe hypoglycemic episodes decreased by $96 \%$, although the statistical significance was reached only at $\mathrm{T} 1$ and not at $\mathrm{T} 2$.

It can be reasonably presumed that the reduction in the risk of hypoglycemia and the improvement of metabolic control observed with IDeg in this study could play a role in improving patients' quality of life and in reducing the cost burden of hypoglycemia. 
Table 2 Trends over time in continuous endpoints: results of hierarchical linear models

\begin{tabular}{|c|c|c|c|c|}
\hline Outcome & $\begin{array}{l}\text { Time } \\
\text { point }^{a}\end{array}$ & $\begin{array}{l}\text { Estimated mean values } \\
(95 \% \mathrm{CI})\end{array}$ & $\begin{array}{l}\text { Estimated mean change from } \\
\text { baseline }(95 \% \mathrm{CI})\end{array}$ & $p$ value \\
\hline \multirow[t]{3}{*}{ HbAlc (\%) } & T0 & $7.97(7.78-8.16)$ & - & - \\
\hline & $\mathrm{T} 1$ & $7.70(7.52-7.88)$ & $-0.27(-0.43$ to -0.11$)$ & $0.001^{*}$ \\
\hline & $\mathrm{T} 2$ & $7.63(7.48-7.78)$ & $-0.34(-0.47$ to -0.21$)$ & $<0.0001^{*}$ \\
\hline \multirow[t]{3}{*}{ Fasting blood glucose $(\mathrm{mg} / \mathrm{dL})$} & T0 & $199.42(191.00-207.84)$ & - & - \\
\hline & $\mathrm{T} 1$ & $176.06(168.47-183.65)$ & $-23.36(-31.84$ to -14.88$)$ & $<0.0001^{*}$ \\
\hline & $\mathrm{T} 2$ & $174.60(166.98-182.22)$ & $-24.82(-34.33$ to -15.31$)$ & $<0.0001^{*}$ \\
\hline \multirow[t]{3}{*}{ Post-prandial glucose $(\mathrm{mg} / \mathrm{dL})$} & T0 & $186.61(177.99-195.23)$ & - & - \\
\hline & $\mathrm{T} 1$ & $165.92(158.49-173.35)$ & $-20.68(-28.71$ to -12.65$)$ & $<0.0001^{*}$ \\
\hline & $\mathrm{T} 2$ & $169.37(161.43-177.31)$ & $-17.23(-27.14$ to -7.32$)$ & $0.001^{*}$ \\
\hline \multirow{3}{*}{$\begin{array}{l}\text { Standard deviation of mean } \\
\text { blood glucose }\end{array}$} & T0 & $80.38(76.95-83.81)$ & - & - \\
\hline & $\mathrm{T} 1$ & $75.24(72.06-78.42)$ & $5.14(-7.09$ to -3.19$)$ & $<0.0001^{*}$ \\
\hline & $\mathrm{T} 2$ & $74.71(71.53-77.89)$ & $-5.67(-8.43$ to -2.91$)$ & $<0.0001^{*}$ \\
\hline \multirow[t]{3}{*}{ HBGI } & T0 & $13.62(11.88-15.36)$ & - & \\
\hline & $\mathrm{T} 1$ & $9.70(8.41-10.99)$ & $-3.92(-5.16$ to -2.68$)$ & $<0.0001^{*}$ \\
\hline & $\mathrm{T} 2$ & $9.85(8.62-11.08)$ & $-3.77(-5.15$ to -2.39$)$ & $<0.0001^{*}$ \\
\hline \multirow[t]{3}{*}{ LBGI } & T0 & $1.67(1.34-2.00)$ & - & \\
\hline & $\mathrm{T} 1$ & $1.96(1.63-2.29)$ & $0.29(-0.02$ to -0.60$)$ & 0.06 \\
\hline & $\mathrm{T} 2$ & $1.68(1.37-1.99)$ & $0.01(-0.27$ to -0.29$)$ & 0.92 \\
\hline \multirow[t]{3}{*}{ Weight (kg) } & T0 & $71.01(69.11-72.91)$ & - & - \\
\hline & $\mathrm{T} 1$ & $70.73(68.79-72.67)$ & $-0.28(-0.74$ to 0.18$)$ & 0.24 \\
\hline & $\mathrm{T} 2$ & $70.83(68.84-72.82)$ & $-0.18(-0.78$ to 0.42$)$ & 0.56 \\
\hline
\end{tabular}

${ }^{*}$ Estimated mean change from baseline is statistically significant at $p<0.05$

CI Confidence interval, HbAlc glycated hemoglobin, HBGI high blood glucose index, LBGI low blood glucose index

a T0, Baseline; T1, first follow-up visit [median 4.2 (IQR 3.1-5.7) months after T0]; T2, second follow-up visit [median of 9.5 (IQR 7.7-11.3) months after T0]

Furthermore, in our real-world cohort, body weight was unchanged, while in the BEGIN studies it increased slightly in the participants. This result could reflect the reduction in both basal and short-acting insulin doses recorded during the follow-up.

Our data may also be compared with findings from other observational studies. In a European multicenter, retrospective, chart review study (EU-TREAT study) involving 1717 patients with T1DM after 12 months from the initiation of IDeg, HbA1c decreased significantly by $-0.22 \%(2.4 \mathrm{mmol} / \mathrm{mol} ; p<0.001)$ and FBG decreased by $-21.02 \mathrm{mg} / \mathrm{dL}$ $(1.17 \mathrm{mmol} / \mathrm{L} ; p<0.001)$. Total daily insulin dose decreased by $-11 \%$, daily basal insulin dose by $-12 \%$ and daily prandial insulin dose by $-7 \%$. At the 6-month comparison, the switch to IDeg had resulted in significantly lower rates of overall hypoglycemia (21\% 
Table 3 Changes in basal and short-acting insulin dose (IU) during the study

\begin{tabular}{llllr}
\hline Insulin & Time point $^{\mathbf{a}}$ & $\begin{array}{l}\text { Estimated mean } \\
\text { values }(\mathbf{9 5 \%} \text { CI })\end{array}$ & $\begin{array}{l}\text { Estimated mean change } \\
\text { from RC }(\mathbf{9 5 \%} \text { CI })\end{array}$ & $\boldsymbol{p}$ value \\
\hline Basal insulin & T-1 $(\mathrm{RC})^{\mathrm{b}}$ & $21.0(19.6-22.4)$ & - & \\
& T0 & $19.2(17.9-20.5)$ & $-1.8(-2.2$ to -1.4$)$ & $<0.0001^{*}$ \\
& T1 & $19.7(18.4-21.0)$ & $-1.4(-2.1$ to -0.7$)$ & $0.0002^{*}$ \\
Short-acting insulin & T2 & $19.7(18.4-21.0)$ & $-1.4(-2.1$ to -0.7$)$ & $0.0004^{*}$ \\
& T-1 & $27.3(25.6-29.0)$ & - & \\
& T0 & $26.3(24.8-27.8)$ & $-1.0(-1.6$ to -0.4$)$ & $0.002^{*}$ \\
& T1 & $24.3(22.6-26.0)$ & $-2.9(-3.9$ to -1.9$)$ & $<0.0001^{*}$ \\
& T2 & $24.1(22.5-25.7)$ & $-3.1(-4.2$ to -2.0$)$ & $<0.0001^{*}$ \\
\hline
\end{tabular}

${ }^{*}$ Estimated mean change from baseline is statistically significant at $p<0.05$

$R C$ Reference class

a T-1 refers to the last prescribed dose of the previous basal insulin before initiation of insulin degludec (IDeg) treatment

b Mean dose of the previous basal insulin

Table 4 Incidence rate of hypoglycemic episodes during the follow-up

\begin{tabular}{llll}
\hline $\begin{array}{l}\text { Time } \\
\text { point }\end{array}$ & $\begin{array}{l}\text { Nocturnal } \\
\text { hypoglycemic } \\
\text { episodes }\end{array}$ & $\begin{array}{l}\text { Severe } \\
\text { hypoglycemic } \\
\text { episodes }\end{array}$ & $\begin{array}{l}\text { Overall } \\
\text { hypoglycemic } \\
\text { episodes }\end{array}$ \\
\hline T0 & $1.2(0.8-1.9)$ & $0.4(0.2-0.7)$ & $9.1(7.1-11.7)$ \\
T1 & $0.9(0.6-1.3)$ & $0.0(0.0-0.2)^{*}$ & $7.9(6.3-9.9)$ \\
T2 & $0.6(0.4-0.9)^{*}$ & $0.0(0.0-0.5)$ & $5.5(4.3-6.9)^{*}$ \\
\hline
\end{tabular}

Data is reported as the number of hypoglycemic episodes per patient-month with the $95 \% \mathrm{CI}$ given in parenthesis ${ }^{*}$ Estimated mean change from baseline is statistically significant at $p<0.05$

reduction), overall non-severe hypoglycemia (19\% reduction), nocturnal non-severe hypoglycemia (46\% reduction) and severe hypoglycemia (85\% reduction) post-switch versus pre-switch. The results from the 12-month postswitch versus pre-switch comparisons were similar. Body weight had increased by $0.58 \mathrm{~kg}$ at 6 months compared with baseline $(p<0.001)$ and was stable at 12 months ( $p=$ not significant vs. 6 months). No data were collected on glycemic variability [13].

Data from the German/Austrian diabetes database (DPV registry) on 360 patients with
T1DM showed that IDeg was associated with no differences in HbA1c after 3-15 months. The BMI increased from 23.2 to $24.0 \mathrm{~kg} / \mathrm{m}^{2}$ in patients with T1DM who had switched to IDeg $(p<0.0001)$. This was observed in all subgroups with the exception those with prior insulin NPH use [14].

Finally, the safety of IDeg was documented in a study on hospitalized, mainly elderly patients [15].

The major strengths of the study are its multicenter nature, the sample size, the duration of the follow-up compared to the available observational studies, the quality of the data despite their routine care nature and the comprehensive set of endpoints, particularly the data on glycemic variability, PPG and hypoglycemia episodes collected by downloading glucose meters routinely used by the patients.

Among the limitations, the lack of a comparator arm should be mentioned. Participants probably performed a different number of blood glucose measurements according to the different adherence to self-monitoring of blood glucose protocols; this could give heterogeneous estimates because some patients could have a low, or conversely a high, number of measurements. 


\section{CONCLUSIONS}

In conclusion, this real-world study documented that switching patients with T1DM to IDeg from other basal insulins significantly improves glycemic control, reduces glycemic variability and decreases the risk of hypoglycemia, without weight gain.

\section{ACKNOWLEDGEMENTS}

Funding. This was a non-profit, investigators-initiated study. The journal's Rapid Service fee was paid by AIRON Communication, funded through a Novo Nordisk S.p.A. unconditional grant.

Editorial Assistance. Editorial assistance was provided by Paolo De Gennaro and Karin Schlusnus of Airon Communication S.r.l. through a Novo Nordisk S.p.A. unconditional grant.

Authorship. All named authors meet the International Committee of Medical Journal Editors (ICMJE) criteria for authorship for this manuscript, take responsibility for the integrity of the work as a whole, and have given final approval to the version to be published.

Disclosures. Paola Ponzani: Novo Nordisk, GSK, Jansen, Bayer and Novartis for research grants; Eli Lilly for honoraria for speaking and position on advisory board. Cesare Berra: consulting fees from Novo Nordisk, Lilly, Boehringer Ingelheim, Sanofi, Johnson \& Johnson and Bayer; research support from AstraZeneca and Takeda; member of advisory board for Lilly, Novo Nordisk, Boehringer Ingelheim, Sanofi and AstraZeneca; investigator in clinical trials sponsored by Lilly, Sanofi and Novo Nordisk. Chiara Di Loreto: research support from Roche diagnostics. Maria Chiara Rossi: research support from ForaCare, Artsana, Sanofi, Novo Nordisk, Dexcom, Medtronic, AstraZeneca. Alessandra Di Lelio, Paola Del Sindaco, Francesco Reggiani and Giuseppe Lucisano have nothing to disclose.
Compliance with Ethics Guidelines. All procedures followed were in accordance with the ethical standards of the responsible committee on human experimentation (institutional and national) and with the Helsinki Declaration of 1964, as revised in 2013. Informed consent was obtained from all patients for being included in the study. The study protocol was approved by the following Ethics Committees of the participating centers: Comitato Etico Regionale della Liguria, Genova (GE); Comitato Etico Indipendente Istituto Clinico Humanitas, Rozzano (MI); CER UMBRIA, Comitato Etico Regionale Umbria, Perugia (PG).

Data Availability. The datasets analyzed during the current study are available from the corresponding author on reasonable request.

Open Access. This article is distributed under the terms of the Creative Commons Attribution-NonCommercial 4.0 International License (http://creativecommons.org/licenses/ by-nc/4.0/), which permits any noncommercial use, distribution, and reproduction in any medium, provided you give appropriate credit to the original author(s) and the source, provide a link to the Creative Commons license, and indicate if changes were made.

\section{REFERENCES}

1. European Medicines Agency. Insulin degludec. Summary of product characteristics. 2013. http:// www.ema.europa.eu/docs/en_GB/document_library/ EPARProduct_Information/human/002498/WC5001 38940.pdf. Accessed Nov 2019.

2. Heller S, Buse J, Fisher M, et al. Insulin degludec, an ultra-longacting basal insulin, versus insulin glargine in basal-bolus treatment with mealtime insulin aspart in type 1 diabetes (BEGIN Basal-Bolus Type 1): a phase 3, randomised, open-label, treat-to-target non-inferiority trial. Lancet. 2012;379:1489-97.

3. Mathieu C, Hollander P, Miranda-Palma B, et al. "Efficacy and safety of insulin degludec in a flexible dosing regimen vs insulin glargine in patients with type 1 diabetes (BEGIN: Flex T1): a 26-week randomized, treat-to-target trial with a 26-week 
extension. J Clin Endocrinol Metab. 2013;98:1154-62.

4. Davies MJ, Gross JL, Ono Y, et al. Efficacy and safety of insulin degludec given as part of basal-bolus treatment with mealtime insulin aspart in type 1 diabetes: a 26-week randomized, open-label, treatto-target non-inferiority trial. Diabetes Obes Metab. 2014;16:922-30.

5. Heise T, Hermanski L, Nosek L, Feldman A, Rasmussen S, Haahr H. Insulin degludec: four times lower pharmacodynamic variability than insulin glargine under steady-state conditions in type 1 diabetes. Diabetes Obes Metab. 2002;14:859-64.

6. Cryer PE. Hypoglycaemia: the limiting factor in the glycaemic management of type 1 and type 2 diabetes. Diabetologia. 2002;45:937.

7. Wild D, von Maltzahn R, Brohan E, Christensen T, Clauson P, Gonder-Frederick L. A critical review of the literature on fear of hypoglycemia in diabetes: implications for diabetes management and patient education. Patient Educ Couns. 2007;68:10-5.

8. de Lusignan S, Crawford L, Munro N. Creating and using real-world evidence to answer questions about clinical effectiveness. J Innov Health Inf. 2015;22:368-73.

9. Garrison LP Jr, Neumann PJ, Erickson P, Marshall D, Mullins CD. Using real-world data for coverage and payment decisions: the ISPOR Real-World Data Task Force report. Value Health. 2017;10:326-35.
10. Tentolouris N, Knudsen ST, Lapolla A, et al. Switching "Real-World" diabetes patients to degludec from other basal insulins provides different clinical benefits according to their baseline glycemic control. Adv Ther. 2019;36:1201-10.

11. Lualdi C, Silverii A, Dicembrini I, et al. Adjustment of insuline doses when switching from glargine 100 $\mathrm{U} / \mathrm{ml}$ or detemir to degludec: an observational study. J Endocrinol Invest. 2019;42:319-26.

12. Ponzani P, Berra C, Di Lelio A, et al. Impact of insulin degludec in type 2 diabetes: real-world data on effectiveness and safety. Diabetes Ther. 2018;9:2209-18.

13. Siegmund T, Tentolouris N, Knudsen ST, et al. A European, multicentre, retrospective, non-interventional study (EU-TREAT) of the effectiveness of insulin degludec after switching basal insulin in a population with type 1 or type 2 diabetes. Diabetes Obes Metab. 2018;20:689-97.

14. Bohn B, Zimmermann A, Wagner C, et al. Real-life experience of patients starting insulin degludec. A multicenter analysis of 1064 subjects from the German/Austrian DPV registry. Diabetes Res Clin Pract. 2017;29:52-8.

15. Simioni N. Inpatient hyperglycemia management: the opportunities of a new basal insulin. Ital J Med. 2016;10:103-10. 